\title{
Who pays for community care? The same old question
}

\author{
Paul Lelliott, Andrew Sims, John Wing
}

Trends in mental health service funding over the past 40 years show that the programme of hospital closures has not resulted in a significant release of resources to fund community based services. Far from being excessive, the current provision of residential services (both NHS and non-NHS) for mentally ill people is now below levels recommended as sufficient by the government, the Royal College of Psychiatrists, and the National Schizophrenia Fellowship. What clinical research evidence there is suggests that more rather than fewer residential places are required. This situation is likely to be compounded by the recent transfer of responsibility for funding private and voluntary residential care from the Department of Social Security to local authority social services departments.

This paper reviews trends in expenditure on mental health services in England over the past 40 years and discusses the current level of provision of and need for residential facilities for mentally ill people. The historical perspective sounds a warning to planners about the difficulties of diverting funds from hospital to community based services-a warning that is timely in the light of the recent changes in responsibility for those requiring residential care in the community.

Raftery commented recently that mental health services "have been subject to more change than virtually any other type of health service over the past four decades." Services in the United Kingdom are now moving into the third stage of a progressive shift in the locus of patient care. The first stage arose from the decision in the 1950 s to reduce NHS bed numbers and close large psychiatric hospitals. The second, which reached its peak in the 1970s and 1980s, was the development of small local psychiatric units, often on district general hospital sites, with a limited range of community services to meet the residential needs of those who no longer required hospital accommodation. During this period the recommendations of the Seebohm report resulted in separation of responsibility for social work, which became the responsibility of local authority social services departments. ${ }^{2}$ This, together with the incorporation of the medical duties of local authorities into the NHS, led to a split between health and social functions-which hitherto had been interactive. The third stage was heralded in April 1993 by the transfer of responsibility for the funding of many of those living in voluntary sector and private residential homes and hostels from the Department of Social Security to local authority social services departments. This diversion of funds, coupled with the vesting of responsibility for community care in social services departments, is intended to divert people from community based residential homes into their own homes.

Before these recent changes the residential costs of many of those in community based facilities were met by social security benefits. This arrangement was an incentive to local and health authorities both to use and to encourage the development of voluntary and private sector hostels and group homes. It contributed to the more than fourfold increase in the numbers of such facilities during the 1980s (from 2015 in 1978-9 to 8370 in 1989-90). Nevertheless, this period also saw the total number of places in all forms of residential provision for mental illness (NHS psychiatric beds, local authority hostels, and voluntary and private sector facilities) fall by $28 \%$-from 94607 to $68086 .^{3}$

This third stage started well before the second was complete and, unfortunately, is likely to strike a familiar rock: the unresolved issue of where the funds will come from to support the mentally ill in the community.

\section{Trends in the funding of mental health services NATIONAL HEALTH SERVICE}

Between 1958 and 1979, the period of the most rapid bed closures, ${ }^{4}$ the percentage of the NHS hospital and community health services budget spent on mental illness fell from just over $15 \%$ to just over $11 \%$. Since 1979 the percentage of the budget spent on mental illness has remained fairly onstant (table I); in 1990-1 (the most recent year for which there are figures) it was $11 \cdot 8 \%$. (The slight increase since $1988-9$ is due to the inclusion of the mental illness component of comTABLE I-Hospital and community health services expenditure on mental illness services $(f, m, 1990-1 \text { prices })^{5}$

Royal College of Psychiatrists, London SW1X 8PG Paul Lelliott, deputy director research unit

Andrew Sims, past president John Wing, director, research unit

Correspondence to: Dr Lelliott.

BMF 1993;307:991-4

\begin{tabular}{|c|c|c|c|c|c|c|c|c|}
\hline & Inpatient & Outpatient & Day patient & $\begin{array}{c}\text { Total } \\
\text { hospital }\end{array}$ & Community & $\begin{array}{l}\text { Total hospital } \\
\text { and } \\
\text { community }\end{array}$ & $\begin{array}{l}\text { Hospital } \\
\text { provision as \% } \\
\text { of total } \\
\text { hospital and } \\
\text { community } \\
\text { spending }\end{array}$ & $\begin{array}{l}\text { All provision } \\
\text { (including } \\
\text { community) as \% of } \\
\text { total hospital and } \\
\text { community } \\
\text { spending }\end{array}$ \\
\hline $1980-1$ & $1390 \cdot 4$ & 73.5 & $80 \cdot 7$ & $1544 \cdot 6$ & & & $11 \cdot 2$ & \\
\hline $1981-2$ & 1410.9 & 86.8 & $87 \cdot 4$ & $1585 \cdot 1$ & & & $11 \cdot 3$ & \\
\hline $1982-3$ & $1403 \cdot 4$ & 93.6 & $88 \cdot 2$ & $1585 \cdot 2$ & & & $11 \cdot 3$ & \\
\hline $1983-4$ & 1398.0 & 93.0 & $98 \cdot 2$ & $1589 \cdot 2$ & & & $11 \cdot 2$ & \\
\hline $1984-5$ & $1402 \cdot 0$ & 96.6 & $102 \cdot 0$ & $1600 \cdot 6$ & & & $11 \cdot 3$ & \\
\hline $1985-6$ & $1390 \cdot 7$ & $100 \cdot 2$ & $107 \cdot 4$ & $1598 \cdot 3$ & & & $11 \cdot 2$ & \\
\hline $1986-7$ & $1384 \cdot 1$ & $99 \cdot 0$ & 110.4 & 1593.5 & & & $11 \cdot 1$ & \\
\hline $1987-8$ & $1464 \cdot 7$ & $85 \cdot 9$ & 113.6 & $1664 \cdot 2$ & & & 11.5 & \\
\hline $1988-9$ & $1458 \cdot 2$ & $78 \cdot 2$ & $123 \cdot 1$ & 1659.5 & 139.0 & $1789 \cdot 5$ & 11.4 & $12 \cdot 4$ \\
\hline $1989-90$ & $1436 \cdot 1$ & $81 \cdot 4$ & $121 \cdot 4$ & 1638.9 & $167 \cdot 3$ & $1806 \cdot 2$ & $11 \cdot 1$ & $12 \cdot 2$ \\
\hline $1990-1$ & $1365 \cdot 8$ & $84 \cdot 6$ & 128.5 & 1578.9 & 184.6 & 1763.5 & $10 \cdot 6$ & 11.8 \\
\hline
\end{tabular}


munity services.) Recently there has been a slight reduction in the amount spent on hospital care (from $11.5 \%$ of the total hospital and community health services budget in $1987-8$ to $10.6 \%$ in 1990-1). Despite this, and further bed closures, $90 \%$ of the NHS mental illness budget is still spent on hospital based facilities.

The shift of expenditure away from mental illness during the period of maximal bed closures suggests that the dowry that was supposed to have come from the closed asylums to the new community services was never in fact transferred.

There is evidence, particularly from inner city districts, ${ }^{6}$ that patients currently occupying psychiatric beds do need intensive nursing owing to high levels of disability or disturbed behaviour. This probably accounts for the increased staff:patient ratios and therefore the increased unit cost of caring for psychiatric inpatients, which has compounded the lack of the dowry for community care.

\section{SOCIAL SERVICES}

During the 1980s the proportion of personal social services spending on mental illness rose from $1.9 \%$ to $2 \cdot 4 \%$; in $1990-1$ expenditure was about $£ 101 \mathrm{~m}$. Despite this increase, personal social services expenditure on mental illness remains only a fraction (about $5 \%$ ) of that by the NHS. In recent years the mental illness specific grant has made a small extra contribution $(£ 31.4 \mathrm{~m}$ in $1992-3$ - equivalent to an additional $1.5 \%$ of total NHS and personal social services expenditure on mental illness).

\section{Current provision of mental health residential services}

This review of past trends suggests that further bed closures are unlikely to release sufficient resources for community care. Furthermore, two recent surveys suggest that, far from overprovision, there is in fact already an insufficiency of residential care facilities (both NHS and non-NHS) for the mentally ill. These surveys give estimates of the number and type of current provision that are far more detailed, and almost certainly more accurate, than those provided by centrally collected national statistics. They allow comparisons to be made between actual provision and that recommended by the Department of Health, the Royal College of Psychiatrists, and the National Schizophrenia Fellowship.

The first survey, conducted by the Royal College of Psychiatrists' research unit as part of a national audit of new long stay psychiatric patients, covered 48 English districts. It found that the average number of NHS beds available to adult psychiatric patients (those aged 18-64) was 59 per 100000 total population. This total included 30 beds per 100000 that might be considered "residential"- that is, rehabilitation, continuing care, or "ward in a house" accommodation. The remainder were acute and intensive care beds. In addition to this NHS provision, a total of 46 residential places were provided for the adult mentally ill per 100000 by all other agencies (local authorities and the voluntary and private sector; table II).

The second survey, of 35 British health districts and health boards, was undertaken by Research and Development for Psychiatry. ${ }^{7}$ It reported that residential provision (which excluded hospital beds, family placement schemes, and supported lodgings) totalled 37 places per 100000 total population. This figure is identical to the equivalent count from the first survey.

An estimate derived from these data suggests that the current national average of available places totals 105 per 100000 population, broken down into 27 acute hospital beds; two intensive care beds; 67 places in medium and long stay residential provision (hospital ward, hostel, and group home, including non-hospital accommodation); and nine places in family placement schemes and supported lodgings.

\section{Comparison with recommended levels of provision}

In 1985 the government recommended that district general hospital units should on average have 30-50 general psychiatry admission (acute) beds per 100000 population. ${ }^{8}$ Even allowing for the fact that this figure $\stackrel{0}{7}$ included a few beds for the elderly with functional mental illness who require brief admissions, the current provision of acute beds (27) is very much at the lower end of the recommended range. Current numbers of acute beds are only three quarters of the 36 per on 100000 and medium and long term places (NHS and non-NHS combined) only four fifths of the 90 per 100000 recommended in a recent report by the Royal College of Psychiatrists. ${ }^{9}$ The 46 non-NHS residential places available currently fall far short of the 67-100 recommended by the National Schizophrenia Fellowship. ${ }^{10}$

\section{Clinical evidence for inadequacy of current provision}

Most general psychiatrists are aware of the pressures on acute beds caused by high occupancy rates. Two surveys of psychiatric admission units in Greater London, cited in a recent Royal College of Psychiatrists report on facilities and services, ${ }^{11}$ have provided data to support this impression. The first, conducted in 1990 , found that average bed occupancy was $95 \cdot 7 \%^{12}$; in one third of units surveyed it was over $100 \%$ (the Royal College of Psychiatrists recommends that average occupancy should not exceed $85 \%{ }^{8}$ ). The second survey, by Tanner and Pulling, revealed that in some London districts up to one third of acute beds were occupied by mentally ill homeless people. ${ }^{11}$

Evidence that residential services for the long term mentally ill cannot cope with demand is provided by the national audit of new long stay psychiatric patients referred to above. Fifty English districts, serving more than a quarter of the English population, provided information about all patients occupying psychiatric hospital beds who had been admitted within the past three years and had been in hospital continuously for more than six months. Based on these figures, we estimate that there are over 3000 of these severely and chronically disabled "new long stay" hospital patients. Nearly a third of these patients are inappropriately placed in acute wards. The clinicians making the assessments thought that, although nearly two thirds $N$ of the whole sample would be more appropriately placed in a non-hospital setting, only $14 \%$ were capable

TABLE II-Number of NHS beds and non-NHS residential places/ 100000 total population available to psychiatric patients, aged 18-64, in a representative sample of English districts $(n=48)$

\begin{tabular}{lrl} 
& Places/100000 \\
\hline NHS beds & 27 \\
Acute & 2 \\
Intensive care & 9 \\
Rehabilitation & 17 \\
Continuing care & 3 & \\
Ward in a house & 1 & \\
Other & 59 \\
Total & 4 & \\
Non-NHS places & 6 & \\
Unsupported group home & 4 \\
Supported group home & 9 \\
Low staffed hostel & 9 \\
Without sleep in staff & 7 \\
With sleep in staff & 2 \\
High staffed hostel & 5 \\
Supported lodgings & 46 \\
Family placement scheme & \\
Other & \\
Total &
\end{tabular}




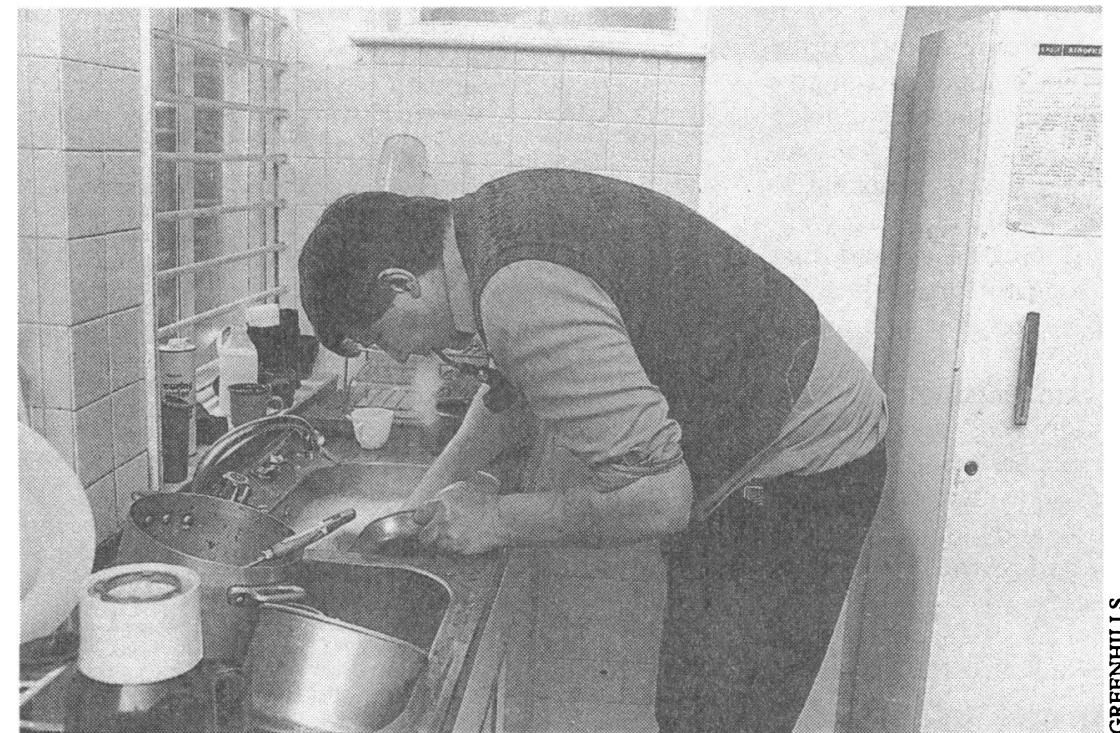

Community facilities may be limited by a vicious circle induced by lack of funds

of living independently or with their families. Nearly one half of those patients who were inappropriately placed could not be transferred either because a suitable facility did not exist or because it was full or would not accept the patient.

These particular patients represent only a fraction of the demand for community based residential accommodation: they occupied only $9 \%$ of all hospital beds. Other groups competing for scarce facilities include so called "revolving door" patients; the homeless mentally ill; patients on acute wards who have problems with accommodation; patients who have been in hospital more than three years (estimated in the new long stay audit to be newly accumulating at a rate of 1.3 per 100000 per year); and mentally ill offenders being transferred back to their district of origin or diverted from custody by the growing number of court diversion schemes.

While there is evidence of unmet need, there is no evidence that residential services are being used wastefully. Thornicroft et al reported that in Southwark only $15 \%$ of recent referrals to residential care could be classed as "inappropriate." 13 The relative dearth of studies on the use of existing residential resources is one area addressed by a project, to be conducted by the Royal College of Psychiatrists research unit, to examine the characteristics and need for care of people in all forms of mental health residential accommodation in eight English districts.

\section{Impact of recent reforms on residential services}

The House of Commons health committee highlighted the paradox at the heart of the white paper Caring for People when it stated, "The new arrangements will operate within the two parameters of cash limits and assessment of need. One side of the equation aims to restrain what has been in the past an open ended commitment to people claiming income support, whilst the other is intended to identify levels of need as never revealed before. This is likely to produce a tension between downward pressure on budgets and increasing demand." 14

The effects of the new legislation on the provision of voluntary and private sector residential accommodation will be compounded by recent curbs on local authority spending. Eighty per cent of directors of social services believed that their budgets would be reduced in April 1993, at precisely the time that they had to take the lead in implementing the NHS and Community Care Act. ${ }^{15}$

There are several implications of this paradox and the new perverse incentives that the legislation may encourage. ${ }^{13}$

Service provision may not meet the need-In effect funding for residential care has been capped. The overriding incentive for care managers with responsibility for assessing patients will be to stay within budget. The system being replaced, with its openended financial commitment, was at least driven by demand. There is little evidence that this led to unnecessary or wasteful use of residential care by the mentally ill. Indeed, Griffiths himself, the architect of the reform, stated, "By international comparisons we do not as yet have excessive numbers of people in residential accommodation."16

Information on unmet need may be suppressed-The fact that local authorities face the threat of being sued for identifying needs that they cannot then meet has stimulated a debate on whether such information should be made public. It is clearly in the public's interest that it is, otherwise the assessment process will not be able to help service planning or allow for the aggregation of data to produce epidemiologically based estimates of the need for residential care.

There are no incentives to discharge severely mentally ill people from hospital-It has been left to local services to agree what constitutes a need for primarily social as opposed to primarily medical care. While the cash limited budget for providing much of the community based residential provision is held by local authorities, this is likely to be an uneven debate. Care managers may judge that a patient who is in hospital is having his or her needs met, especially if the budget is insufficient and the patient incapable of living independently. There is a clear financial disincentive for care managers to facilitate the discharge of such hospital inpatients. If NHS mental health workers assess an inpatient as needing residential care the requirements of the care programme approach will prevent them from discharging that patient until a suitable placement is available. This will slow discharge and prevent it altogether if care managers either disagree with the assessment or are unable to fund the place.

Who will supply the domiciliary care?-If the changes mean that more severely ill patients are returned to their own homes (as opposed to residential settings) there will be increased demand for intensive community support, which, as the review of mental illness funding suggests, could not easily be funded by health authorities diverting resources away from hospital based services. Will fundholding general practices have an incentive to purchase community nursing care for patients moved into their own homes instead of a residential setting? There is good evidence that the move over the past decade towards basing community psychiatric nurses in primary care settings has led to them having a much lower proportion of patients with schizophrenia on their caseloads. ${ }^{17}$ Recent increases in the number of fundholding practices may accentuate this process. The conversion of many field social workers into "care managers" has removed large numbers of skilled workers from providing direct care and therefore reduced the ability of social services departments to offer direct care to mentally ill people in their own homes.

\section{Vicious circle}

There is a real danger of a vicious circle being created as discharge from hospital becomes increasingly difficult and admission wards more and more congested. This would itself put pressure on community services as respite, prophylactic, and other planned admissions become impossible. Hard pressed health authority mental health services may have to respond by creating more medium and long stay beds, at the 
expense of further community developments. If existing community services are compromised in this way the circle will be completed as the components of care necessary to allow for the discharge of severely disabled patients, particularly accommodation and regular support, will not be available. Regardless of the extent to which this process occurs, historical trends in expenditure and related service developments suggest that future policy makers cannot look to further hospital bed closures to provide funds to develop community mental health services.

Although social service departments now have the lead responsibility for community care, they still have only a fraction of the total mental health budget. If they discharge their duty to assess individuals' needs for care openly and honestly a simple and familiar question remains over who will pay for and provide the care to meet the need so measured.

1 Raftery J. Mental health services in transition. $\operatorname{Br} \mathcal{F}$ Psychiatry 1992;161: 589-93.

2 Committee on Local Authority and Allied Personal Social Services. Report. London: HMSO, 1968 (Cmnd 3707).

3 Department of Health and Office of Population Censuses and Surveys. The government's expenditure plans 1993-94 to 1995-96. London: HMSO, $1992(\mathrm{Cm} 2212)$.
4 Office of Health Economics. Mental health in the 1990s: from custody to care? London: OHE, 1989. Session 1992-93. London: HMSO, 1993 (HC 489).

6 Patrick M, Higgit A, Holloway F. Changes in an inner city psychiatric inpatient service following bed losses: a follow-up of the East Lambeth 1986 survey. Health Trends 1989;21:121-3.

7 Faulkner A, Field V, Lindesay J. Who is providing what? Information about UK residential care provision for people with mental health problems. London: Research and Development for Psychiatry, 1992.

8 Department of Health and Social Security. Government response to the second report from the social services committee, 1984-85 session. London: HMSO, 1985.

9 Royal College of Psychiatrists. Mental health of the nation: the contribution of psychiatry. London: Royal College of Psychiatrists, 1992.

10 Hammond T, Wallace P. Housing for people who are severely mentally ill. London: National Schizophrenia Fellowship, 1991.

11 Royal College of Psychiatrists. Facilities and services for patients who have chronic persisting severe disabilities resulting from mental illness. London: Royal College of Psychiatrists, 1992

12 Hollander D, Tobiansky R. Crisis in admission beds. BM 1990;301:664.

13 Thornicroft G, Ward P, James S. Care management and mental health. BMF 1993;306:768-71.

14 House of Commons Health Committee. Community care: funding from April 1993. London: HMSO, 1993.

15 Association of Directors of Social Services. ADSS fears that community care "may fail to thrive". London: ADSS, 1993 (press statement 14 Jan).

16 Griffiths R: Community care: an agenda for action. London: HMSO, 1988.

17 Wooff K, Goldberg DP, Fryers T. Patients in receipt of community psychiatric nursing care in Salford 1976-82. Psychol Med 1986;16:407-14.

(Accepted 12 August 1993)

\section{Economic Evaluation and Health Care}

\section{The policy context}

\section{Ray Robinson}

This is the last in a series of articles that describes the ways in which methods of economic evaluation may be used to assess the economic costs and consequences associated with different forms of health care intervention
Institute for Health Policy Studies, Faculty of Social Sciences, The University, Southampton SO9 5NH Ray Robinson, professor of health policy
The final article in the series considers the ways in which the methods described previously are used in the formation of policy. When health authorities are making decisions about how to spend their money they have to draw on several sources of information about priorities: diktats from policy makers, opinions of consumers and of the professional bodies involved, and evidence gained from research. They must also consider the various methods of costing and select the right one for their circumstances. Some of these methods are still in the early stages of development, but more are being developed all the time and they have a valuable role in helping decision making throughout the NHS.

In previous articles I have described the different methods of economic evaluation that are available to assist decision making in the health care sector. Here I consider the ways in which these techniques are and should be used in practice.

\section{Economic evaluation and health care decisions}

The growth in expenditure on health care and the dominance of public sector funding in practically all industrialised countries means that the quest for more cost effective use of limited public sector resources is universal. ${ }^{1}$ I have already described how cost utility analysis was used to devise rules for a more cost effective use of the state's Medicaid budget in the widely publicised Oregon experiment. Elsewhere, Drummond has described how draft guidelines have been drawn up in both Australia and Canada that will require evidence on cost effectiveness to be submitted together with evidence on safety and efficacy as a condition for public reimbursement of the costs of drugs. $^{2}$

In Britain a modified form of economic evaluation, "option appraisal," has been a required component of

\section{Practical steps in setting priorities for purchasing}

- Define programmes

- Estimate programme budgets

- Define sub-programmes

- Identify margins

- Draw up marginal incremental and decremental "wish lists"

- Set priorities

NHS capital planning for a number of years. Within individual programme areas, however, applications of the method in policy contexts are rare. None the less, there are signs that this is changing. For example, it is noticeable that the recently published Department of Health research strategy document, Research for Health, emphasised that henceforth decisions about paying for departmental research and development will be driven by policy problems within the NHS, and in his foreword Professor Michael Peckham pointed out that the lack of information on cost effectiveness was a "real handicap to purchasers and providers of care."

Any strategy that is designed to transfer research on cost effectiveness into practical policy measures needs to be based on an appreciation of the ways in which decisions about policy are actually made. As far as the use of economic evaluation is concerned, the ways in which health authorities as purchasers of care make decisions about how to allocate resources provides a good example.

\section{Health authorities: purchasing and priorities}

Since the implementation of the NHS and Community Care Act in April 1991, district health authorities have acquired new responsibilities as purchasers or 